\title{
Frequency dependence of coda $Q$ : Part I. Numerical modeling and examples from Peaceful Nuclear Explosions
}

\author{
Igor B. Morozov, ${ }^{1}$ Chaoying Zhang, ${ }^{1}$ Joel N. Duenow, ${ }^{2,3}$ Elena A. Morozova, ${ }^{2}$ and Scott B. \\ Smithson ${ }^{2}$ \\ ${ }^{1}$ Department of Geological Sciences, University of Saskatchewan, Saskatoon, SK S7N 5E2, Canada \\ ${ }^{2}$ Department of Geology and Geophysics, University of Wyoming, Laramie, WY 82071, USA \\ ${ }^{3}$ Now at: Colorado School of Mines, Golden, CO 80401, USA
}

\begin{abstract}
Frequency-dependent coda attenuation values are often reported; however such measurements usually depend on the types of the $Q(f)$ models employed. We use numerical modeling of Peaceful Nuclear Explosion (PNE) codas at far regional to teleseismic distances to compare two of such models, namely the conventional frequency-dependent $Q_{\text {coda }}(f)=Q_{0} f^{\eta}$ and frequency-independent coda attenuation $\left(Q_{c}\right)$ with geometrical attenuation $(\gamma)$. The results favor strongly the $\left(\gamma, Q_{c}\right)$ model and illustrate the mechanisms leading to apparent $Q_{\text {coda }}(f)$ dependencies. Tests for variations of the crustal velocity structures show that the values of $\gamma$ are stable and related to lithospheric structural types, and the inverted $Q_{c}$ values can be systematically mapped into the true $S$-wave attenuation factors within the crust. Modeling also shows that $\gamma$ could increase in areas where relatively thin attenuating layers are present within the crust; such areas could likely be related to younger and active tectonics. By contrast, when interpreted by using the traditional $\left(Q_{0}, \eta\right)$ approach, the synthetic coda shows a strong and spurious frequency dependence with $\eta \approx 0.5$, which is also similar to the observations.

Observed $\mathrm{Lg}$ codas from two Peaceful Nuclear Explosions located in different areas in Russia show similar values of $\gamma \approx 0.75 \cdot 10^{-2} \mathrm{~s}^{-1}$, which are also remarkably close to the independent numerical predictions. At the same time, coda $Q_{\mathrm{c}}$ values vary strongly, from 850 in the East European Platform to 2500 within the Siberian Craton. This suggests that parameters $\gamma$ and $Q_{c}$ could provide stable and transportable discriminants for differentiating between the lithospheric tectonic types and ages, and also for seismic coda regionalization in nuclear test monitoring research.
\end{abstract}

\section{Introduction}

Many seismic studies address properties of seismic coda from earthquakes and explosions, and in particular, its amplitude decay and attenuation. Short-period coda amplitude decays lead to the most stable estimates of source magnitudes (Phillips, 2004), and coda attenuation is often related to crustal properties that are difficult to measure otherwise (Aki and Chouet, 1975). High-frequency ( $>5 \mathrm{~Hz}$ ) codas from Peaceful Nuclear Explosions (PNE) in the East European Platform also led several researchers (Ryberg et al., 1995; Enderle et al., 1997; Morozov et al., 1998a,b; Ryberg and Wenzel, 1999; Morozov, 2001) to propose insightful yet sharply contrasting models of small-scale heterogeneity of the uppermost mantle.

Despite widespread observations of frequency-dependent coda-, body-, and surface-wave attenuation, the causes of these frequency dependencies are still not well understood. Frequencydependent $Q$ values are commonly presented in a power-law form: 


$$
Q(f)=Q_{0}\left(f / f_{0}\right)^{\eta}
$$

where $f_{0}$ is some reference frequency often taken to equal $1 \mathrm{~Hz}$, and $Q_{0}$ is the value of $Q$ at this frequency (Aki and Chouet, 1975). $Q(f)$ typically increases with the frequency $(\eta>0)$, and large values of $\eta \sim 0.5$ - 1 are not uncommon in $L g Q$ and $L g$ coda $Q$ studies (e.g., Nuttli, 1973; Hasegawa, 1985; Der et al., 1986; Campillo, 1987, 1990; Steensma and Biswas, 1988; Benz et al., 1997; Mandal and Rastogi, 1998; Frankel et al., 1990; McNamara et al., 1996; Mitchell and Cong, 1998; Mitchell et al., 1997, 1998; McNamara, 2000; and Erickson et al., 2004). Such an increase is somewhat counter-intuitive, considering that the observed abundance of heterogeneities is usually greater at shorter scale-lengths. Positive frequency dependence of $Q(f)$ at short periods led Aki (1980) to advancing the absorption-band hypothesis, and the strong drop in the observed $\eta$ values near $10-\mathrm{Hz}$ frequencies was identified as the "10-Hz transition problem" (Abercrombie, 1998). Visco-elastic rheological models of Earth materials (Liu et al., 1976) and scale-length selective distributions of scale-lengths of scatterers within the crust (e.g., Sato, 1990; Sato and Fehler, 1998) were proposed to explain the observations of the frequency-dependent $Q(f)$. Worldwide data compilations suggest that active tectonic regions are generally characterized by low $Q_{0}$ and high $\eta$, while stable cratons - by higher $Q_{0}$ and lower $\eta$ (e.g., Aki, 1980; Steensma and Biswas, 1988; Benz et al., 1997; Mandal and Rastogi, 1998; Erickson et al., 2004). However, in Peaceful Nuclear Explosion (PNE) studies in the Siberian Craton, extremely high values of $\eta \approx 1$ were also found in combinations with high $Q_{0}$ (Morozov et al., 2006), which appeared to be in conflict with the above trend for stable areas.

The contradiction above indicates the need for re-examining the causes of the $Q(f)$ dependence, and for this we need to look at the initial observations. Note that for $\eta \approx 1$, the amplitude decay rate with time becomes frequency-independent. This means that the amplitude decay is purely geometrical, which potentially could include scattering on large-scale structures (Dainty, 1981). This observation further suggests that also in general, observations of non-zero $\eta$ could in fact be related to lower intrinsic attenuation $\left(Q>Q_{0}\right)$ combined with significant geometrical effects. To quantify these geometrical effects, let us start from the standard model of time-frequency dependent coda amplitudes (Aki and Chouet, 1975):

$$
A(t, f)=A_{0}(f) t^{-\varsigma} \exp \left(-\frac{\pi f}{Q(f)} t\right) .
$$

Here, $t$ is the time after the primary arrival, $A_{0}(f)$ is the coda spectrum at $t=0$, and the factor $t^{-\zeta}$ represents the geometrical spreading of the wave amplitudes which is not compensated by the scattering volume increase with lag time $t$. Usually, values of $0,1 / 2$, or 1 are used for $\zeta$, although Frankel et al. (1990) showed that it could also be variable within different regions. For the farregional and teleseismic PNE codas considered here, $\zeta \approx 0$, corresponding to an approximation of coda waves with cylindrical propagation symmetry (i.e., consisting predominantly of surface or guided waves) and generated near the surface (Morozov and Smithson, 2000). Note that the attenuation parameter $Q(f)$ in eq. (2) still needs to be carefully defined depending on whether factor $t^{-\zeta}$ is considered as fixed or variable.

The power-law dependence (1) is generally dictated by convenience and assumptions about the scaling and symmetry properties of the wavefield (via factor $t^{-\zeta}$ in eq. (2)). Although in many cases this dependence is adequate for fitting the observations, the acute sensitivity of parameters $\left(Q_{0}, \eta\right)$ to the assumed values of $\zeta$ is also well known (e.g., Kinoshita, 1994; Adams and Abercrombie, 1998). As we show below, by exploiting this sensitivity , this ambiguity as well as the frequency dependence of $Q(f)$ can be removed and explained by geometrical effects. 
Compensation of geometrical spreading in equation (2) is relatively accurate for deeper, local earthquake codas involving straight scattered rays and uniform scattering volumes (Aki and Chouet, 1975). However, at shallow depths, crustal-scale and regional distances, scattering tends to concentrate near the surface, and scattered waves consist of complex assemblages of diving, refracted, reflected, guided, and surface modes. Based on various assumptions about these modes and stochastic properties of the crust, several coda models can be used (e.g., Aki and Chouet, 1975; Sato and Fehler, 1998). However, in practice, ad hoc distance and spectral corrections are still required for virtually any model (Mayeda and Walter, 1996; Phillips et al., 2004). Here, we introduce such a correction by noting that an unknown, uncompensated "geometrical attenuation" $G(t)$ should be present in the coda:

$$
A(t, f)=A_{0}(f) t^{-\varsigma} G(t) \exp \left(-\frac{\pi f}{Q(f)} t\right) .
$$

The geometrical attenuation factor $G(t)$ includes both uncompensated geometrical spreading and scattering (elastic attenuation; Dainty, 1981). Because these two contributions can only be separated based on a detailed, well-constrained wave propagation model, such separation could be very difficult for most observations. Nevertheless, for empirical coda characterization, we can lump these two factors together in a single effective geometrical attenuation function $G(t)$.

Because with typical datasets it is only practical to seek a two-parameter coda description (similarly to $\left(Q_{0}, \eta\right)$ in equation (1)), we abandon the frequency dependence of $Q$ by setting $Q(f)=$ $Q_{\mathrm{c}}$ in relation (3) and approximate $G(t)$ as $\exp (-x t)$, obtaining:

$$
A(t, f)=A_{0}(f) t^{-\varsigma} \exp \left[-\left(\gamma+\frac{\pi f}{Q_{\mathrm{c}}}\right) t\right],
$$

where the geometrical attenuation (relaxation) parameter $\gamma$ is measured in units of $1 /$ time. Note that the case of $\eta=1(Q \propto f$ in equation (2)) simply corresponds to a non-zero $\gamma$ and low intrinsic attenuation $\left(1 / Q_{\mathrm{c}}=0\right)$ in relation $(4)$.

In a theoretical analysis of scattering, parameterization (4) was used by Dainty (1981), who showed that at 1-30 Hz, the observed $Q(f)$ of surface waves could be best described by the following expression (Warren, 1972):

$$
\frac{1}{Q(f)}=\frac{1}{Q_{S}}+\frac{g_{0} V_{S}}{2 \pi f},
$$

where $Q_{S}$ is the $S$-wave quality factor for intrinsic attenuation, $V_{S}$ is the $S$-wave velocity, and $g_{0}$ is the turbidity describing the elastic attenuation. Recently, Padhy (2005) also utilized this parameterization to describe the attenuation of high-frequency scattered $L g$ and $S$ waves. If larger scatterers dominate the scattering, $g_{0}$ can be considered constant (Dainty, 1981), and therefore equation (5) becomes equivalent to (4) with $\gamma=g_{0} V_{S} / 2$. Note that for seismic coda, such larger scatterers could effectively be represented by strong velocity gradients, faults, the Moho, sedimentary boundaries, and other crustal structures affecting the propagation of scattered coda waves. An additional theoretical rationale for using form (4) in surface- and body-wave studies was given in Morozov (in press).

Thus, it appears that the $\left(Q_{0}, \eta\right)$ description of frequency-dependent coda attenuation (and maybe also in other types of crustal $Q)$ can be effectively replaced with $\left(\gamma, Q_{c}\right)$. Several advantages arise from such a replacement: 1) formulas (3) and (4) do not rely on wavefield models and assumptions about geometrical spreading or scattering but empirically parameterize 
them instead; 2) geometrical attenuation and $Q_{\mathrm{c}}$ are explicitly separated; and 3) $Q_{\mathrm{c}}$ becomes frequency-independent, in agreement with simple Newtonian solid-state mechanics and at least within the presently available data resolution. The somewhat puzzling increase of coda $Q(f)$ with frequency suggesting depletion of scatterers at shorter scale-lengths becomes explained by geometrical attenuation due to reflected (among other) scattered waves. The geometrical model $\left(\gamma, Q_{c}\right)$ appears to alleviate the " $10-\mathrm{Hz}$ transition problem" and provides a new perspective on the absorption band hypothesis (Morozov, in press). Most importantly, as demonstrated by a worldwide compilation of $\left(\gamma, Q_{c}\right)$ values (Morozov, in press) and by an example from PNE profiles below, parameter $\gamma$ appears to be relatively stable across large areas and correlates with tectonic types and ages. By contrast, $Q_{c}$ shows strong variability, particularly within the activetectonic regions (Morozov, in press).

In this paper, we analyze the $\left(Q_{0}, \eta\right)$ - and $\left(\gamma, Q_{c}\right)$-type models of coda attenuation by using numerical examples and by inverting real PNE coda amplitudes. We generate four finitedifference models of PNE coda wavefields resulting from realistic crustal and upper-mantle velocity models and test them for variations of velocities and $Q$ within the crust. As expected, the $\left(Q_{0}, \eta\right)$ model leads to strong spurious coda $Q(f)$ dependencies. Inversion of this $Q(f)$ for the true crustal attenuation could represent a non-trivial task. By contrast, in the $\left(\gamma, Q_{c}\right)$ model, the values of $\gamma$ can be predicted from the crustal structures by numerical wavefield modeling. Coda $Q_{\mathrm{c}}$ can be uniquely related to the average $S$-wave $Q_{S}$ of the crust, assuming its proportionality to the $P$ wave quality factor $Q_{P}$. Wavefield modeling performed for several types of velocity structures allows us to construct "calibration" dependences $Q_{S}\left(Q_{c}\right)$ and use them to invert the observed coda data for crustal properties in Part II of this study. In addition, a detailed development of the $(\gamma$, $Q_{c}$ ) interpretation, correlation with tectonic types and ages, and comparison to a number of borehole, $L g$, and coda $Q$ studies was presented in Morozov (in press).

\section{Modeling P-wave coda of PNE arrivals}

We examine the effective frequency dependence of coda attenuation by numerically modeling the teleseismic $P$ wave coda resulting from scattering of seismic energy from nearsurface crustal heterogeneities. The use of near-surface crustal scatterers simplifies our computations, and it is also justified by the increased heterogeneity of the shallow crust and supported by several array observations (e.g., Greenfield, 1971; Dainty, 1985 and 1990; Bannister et al., 1990; Gupta et al., 1991) and previous coda modeling (Dainty and Schultz, 1995). The choice of the $P$-wave coda is due to several reasons (Morozov and Smithson, 2000): 1) $S$ - and $L_{g}$ phases are less pronounced in the PNE records; 2) their codas lie on top of the $P$ wave coda, complicating the observation and requiring their decomposition; and 3) $L_{g}$ phase is significantly more difficult to model accurately. Similarly, the Rayleigh wave $\left(R_{g}\right)$ is not examined here because it decays quickly in near-surface sediments and is not viewed as a significant factor in coda formation (Dainty, 1985). $R_{g}$ is also not observed beyond $\sim 200 \mathrm{~km}$ in PNE records. Finally, the use of $P$-wave coda at offsets of $\sim 2900-\mathrm{km}$ provides a sufficient coda time window before the onset of the $S$ wave.

\section{Crustal and coda attenuation}

Three different types of $Q$ values need to be differentiated in this study. First, the intrinsic crustal attenuation describes the loss of seismic energy within the crust, caused by internal friction, friction along grain boundaries, and damping by fluids in cracks and pores. It is described by intrinsic $Q_{P}$ and $Q_{S}$ values corresponding to the $P$ - and $S$-wave energy dissipation during one wave cycle, respectively (Aki and Richards, 2002). For simplicity, we only test crustal models with proportional values of $Q_{P}$ and $Q_{S}$. The second type is our empirical frequency- 
independent quality factor $Q_{c}$ obtained by applying eq. (4) to the spectral slope measurements. The third type of $Q$ is the observed apparent coda quality factor, $Q_{\text {coda }}(f)$. It is based on the same time-frequency amplitude model (2), in which $\zeta$ is set equal to the theoretically-expected level of 0 . From eq. (4): $Q_{\text {coda }}^{-1}(f)=\gamma /(\pi f)+Q_{\mathrm{c}}^{-1}$, and consequently $Q_{\text {coda }}(f)$ is frequency-dependent when $\gamma \neq 0$. This type of $Q$ is most often used in attenuation studies and is commonly approximated in the power-law form (1). However, for reasons explained above, our goal is to return from $Q_{\text {coda }}(f)$ to the stable empirical parameter $Q_{c}$, and from it - to constrain the in situ crustal $Q_{P}$ and $Q_{S}$.

Morozov and Smithson (2000) performed trace envelope measurements in Quartz PNE records for a teleseismic $P$ coda. Although these authors used only two frequency values, the results suggested that $Q_{\text {coda }}(f) \approx 270 f^{0.3}$, where $f$ is expressed in Hz. These estimates were within the range of expected crustal-average $Q_{S}$ values and therefore supported the authors' association of the coda with $L_{g}$ waves scattered within the crust. Here, we attempt to improve on this estimate by using numerical modeling to derive a $Q_{c}\left(Q_{S}\right)$ dependence and re-interpret the observed $Q_{\text {coda }}(f)$ in terms of a frequency-independent $Q_{\mathrm{c}}$ and $\gamma$.

To fully address the crustal scattering problem, 3-D modeling of crustal features including faults, mountain ranges, basins, shorelines, and topography is required. However, full 3-D modeling is still not practical at this time due to limited knowledge of the structural detail and scattering properties of the crust. Instead, we use a heuristic simulation of teleseismic $P$-wave coda amplitude decay modeled as scattering from heterogeneities near the Earth's surface, by combining 1-D reflectivity synthetics with the Born approximation of coda intensity. As this is not a 3-D crustal model, it cannot provide a completely realistic picture of the resultant coda decay; however, it captures the key mechanism of coda generation, provides improved estimates of crustal attenuation, and elucidates the problem of frequency-dependent $Q_{\text {coda }}(f)$.

\section{Numerical Coda Model}

Our scattering model represents crustal heterogeneities as point scatterers distributed within the Earth's crust. The resultant coda intensity (energy density) $U$ recorded at a receiver at time $t$ is an integral over volume $V$ containing all scatterers:

$$
U(\vec{r}, t)=\int d t_{s} \iiint_{V} d^{3} \vec{r}_{s} \Psi\left(\vec{r}_{s}\right) U_{\text {source }}\left(\vec{r}_{s}, t_{s}\right) G\left(\vec{r}_{s}, t_{s} ; \vec{r}, t\right)
$$

where $t_{s}$ is the time of the direct arrival at the scatterer, $\vec{r}_{s}$ represents the scatterer positions, $\Psi$ is the scattering potential describing the amount of energy reflected at each scatterer, $U_{\text {source }}$ is the seismic source function describing the energy density arriving at the scatterer from the source, and $G(\ldots)$ is the Green's function, describing the propagation of scattered energy from the scatterer to receiver. Each scattering point acts as a secondary source for seismic waves (Figure 1). Because the upper crust is assumed to be the primary contributor to the seismic coda, this volume integral is further replaced with a surface integral:

$$
U(\vec{r}, t)=\int d t_{s} \iint_{S} d^{2} \vec{r}_{s} \Psi\left(\vec{r}_{s}\right) U_{\text {source }}\left(\vec{r}_{s}, t_{s}\right) G\left(\vec{r}_{s}, t_{s} ; \vec{r}, t\right) \text {. }
$$

This approximation also allows us to reduce the volume of simulations by considering only the surface-to-surface Green's functions and to use a near-surface source modeling algorithm.

The Green's function $G$ is translationally invariant in time and space: 
$G\left(\vec{r}_{s}, t_{s} ; \vec{r}, t\right)=G\left(\vec{r}-\vec{r}_{s}, t-t_{s}\right)$,

and therefore:

$U(\vec{r}, t)=\int d t_{s} \iint_{S} d^{2} \vec{r}_{s} \Psi\left(\vec{r}_{s}\right) U_{\text {source }}\left(\vec{r}_{s}, t_{s}\right) G\left(\vec{r}-\vec{r}_{s}, t-t_{s}\right)$.

For a given coda time $t$ and velocity $v=\frac{\left|\vec{r}-\vec{r}_{s}\right|}{\left|t-t_{s}\right|}$ chosen for a particular scattered mode (e.g., $v=2.9-3.5 \mathrm{~km} / \mathrm{s}$ for a scattered $L_{g}$ ), the contributions of scattered energy originate from an elliptical ring surrounding the receiver (Morozov and Smithson, 2000). The scattering area within the ring increases with time due to its increasing radius and partly compensates the energy decay due to the geometrical attenuation. For example, for a plane of constant scattering potential and no intrinsic attenuation, the coda energy would stay constant, provided that only surface-wave modes contribute to the Green's function (8).

In equation (7), by using the primary field as $U_{\text {source }}\left(\vec{r}_{s}, t\right)$, we only consider receiver-end scattering, similarly to how it was done in Morozov and Smithson (2000). Due to reciprocity, the same coda should be produced by near-source scattering in the 1-D model. Dainty (1990) showed that this was also true in real records from Semipalatinsk nuclear explosions recorded at NORESS. In the single-scattering approximation of this study, the powers of the source- and receiver-end codas will add up, and the resulting dependence of coda slopes on the frequency should not change.

Inclusion of the near-source scattering would double the resulting singly-scattered coda intensity. However, in our approach, this intensity is controlled by factor $\Psi$ in equation (9), which is set equal to a frequency-independent constant for simplicity. The value of this constant was established by matching the modeled coda amplitudes to those observed from PNE Quartz-4 (Morozov and Smithson, 2000; also see Discussion below), and consequently it incorporates the contributions from both source and receiver areas. Therefore, the results of modeling using equations (7-9) should be valid for single shallow scattering approximation of both near-source and near-receiver scattering.

\section{Synthetic Green's functions}

Synthetic seismic sections created using the reflectivity method (Fuchs and Müller, 1971) were used as source and Green's functions in several 1-D crustal and upper mantle models (Figure 2). The original FORTRAN program by K. J. Sandmeier was modified to handle larger computations, parallelized, and incorporated into our data processing system (now called IGeoS; Chubak and Morozov, 2006) allowing seamless filtering, inverting, saving, and plotting the results. A detailed description of this tool can be obtained from the following link: http://seisweb.usask.ca/igeos/cs.php?doc=reflect.

Computations were performed on a 66-processor 1.6-GHz AMD Opteron cluster and produced 800 -second, 3-component synthetic records sampled at 20-ms intervals and output at $10-\mathrm{km}$ intervals from near-zero to $3500-\mathrm{km}$ distances from the source. Modeling frequency band was $0.2-20 \mathrm{~Hz}$ using a "delta-function" source function suitable for spectral measurements. The frequency band, listening times, and offset ranges were selected to cover the corresponding parameter ranges in PNE recordings (e.g., Morozova et al., 1999). Sufficiently dense phase velocity spectrum was selected in order to avoid frequency aliasing during numerical mode summations. As with any implementations of the propagator matrix method (Aki and Richards, 2002), all P/SV mode conversions and multiples were accounted for in the modeling. 
We used four different velocity models to compute the synthetics (Figure 2), including the global IASP91 model (Kennett and Engdahl, 1991) consisting of a simple three-layer crust and a mantle without strong gradients and low-velocity zones. However, the IASP91 model is too slow for the platform areas of northern Eurasia, and the remaining three models were based on the detailed models derived from the NE profile Quartz and considered the best match for the study area (Mechie et al., 1993; Morozova et. al., 1999). The "Quartz" model contains a 3-gradient layer crust overlain by a 3-km-thick sediment layer and complex mantle with low velocity zones at 110- and 210-km depths (Figure 2a). Another, "Complex Crust" model has a high-contrast, 5layer crust with a somewhat exaggerated attenuative layer $\left(Q_{S}=10\right)$ on its top and the Quartz mantle (Figure 2b). Model "Warm Quartz" (Figure 2a) was an attempt to simulate a high-heat flow regime in the Quartz model by applying a temperature-related negative velocity gradient within the crust (Christensen and Mooney, 1995). Note that compared to the IASP91 model, all three Quartz-based models show more complex waveforms containing crustal $L_{g}$ phases in addition to the $P$ and $S$ phases (Figure 3). In order to collect data on the sensitivity of the results to crustal velocities alone, we also repeated the Quartz simulations after applying $\pm 5 \%$ perturbations of crustal velocities (Figure 2a).

Multiple simulations were performed for each model, with $P$ - and $S$-wave quality factors for layers in the crustal part of the model set proportional: $Q_{P}=2 Q_{S}$, with values of $Q_{S}$ ranging from 100 to 1000 to encompass the expected Earth's whole-crust $Q_{S}$ values. This range of $Q_{S}$ corresponds to $L g Q$ values of $\sim 300-1000$ measured from 19 Russian PNEs by Li et al. (submitted to BSSA) and extended to low- $Q$ values in order to investigate the crustal effects. Mantle attenuation in each velocity model was fixed by using the mantle $Q_{p}$ values derived from $P$-wave Quartz PNE data. These $Q_{s}$ values ranged from $Q_{S}=750$ below the Moho to 600 above the 410$\mathrm{km}$ discontinuity, with an attenuative zone of $Q_{S}=200$ in the region of the Lehmann discontinuity at 110-150-km depths (Morozov et. al., 1998b).

As in Morozov and Smithson (2000), the P-wave coda was measured at teleseismic, $2900-\mathrm{km}$ offsets to allow a significant time interval for measurements before the onsets of $P_{g}$ and $S$ waves. Since $L_{g}$ waves originating from the source (or $L_{g}$ waves scattered near the source) travel too slowly to arrive within this time interval, the scattering region was assigned around the receiver only. The region size was chosen to be a 1200-km square on a side, allowing $600 \mathrm{~km}$ from the central receiver to the nearest edges (Figure 4). For scattered waves of $3-\mathrm{km} / \mathrm{s}\left(L_{g}\right)$ velocity, this gives a $\sim 200$-s window in which to reliably measure the coda amplitude decays. As the $Q_{S}$ value in the reflectivity synthetics is constant throughout the crustal section, and crustal scattering does not depend on the source-receiver offsets, the receiver's offset value should not influence $Q_{\text {coda }}(f)$ and $Q_{\mathrm{c}}$ values measured from the output traces.

To obtain seismic traces at appropriate source-scatterer and scatterer-receiver offset distances for use in the numerical convolution (equation (9)), we used a $\tau$ - $p$ wavefield interpolation algorithm (Yilmaz, 1987) using the synthetic seismic sections. The interpolation code was also implemented as an independent tool (see http://seisweb.usask.ca/igeos/cs.php?doc=interp) and was tested separately in other applications.

Numerical evaluation of integral (9) was performed by using Monte-Carlo sampling in order to avoid spurious coherency that could be caused by spatial aliasing when using a regular integration grid. The 2-D spatial integral uniformly samples surface points on a random grid simulating constant scattering potential for the entire infinite plane (Figure 4). Sixty-four realizations of the random grid, each with 2000 points (Figure 4), were computed in parallel on the cluster, and the resulting amplitude envelopes were stacked afterwards to produce the final synthetic coda records (Figure 5). As above, the synthetic coda generation was implemented in an IGeoS tool sharing many of its libraries with other codes 
(http://seisweb.usask.ca/igeos/cs.php?doc=syncoda).

Using the resulting coda records, log-amplitude slope measurements were performed on coda envelopes in the manner of Morozov and Smithson (2000) (Figure 5). $\mathrm{L}_{2^{-}}$(RMS) and $\mathrm{L}_{1^{-}}$ norm fitting (e.g., Aster et. al.; 2002) were tried, with uncertainties measured by using 50-\% jackknife resampling. Owing to large sampling volumes and stable coda envelopes, the differences between the $\mathrm{L}_{2}$ - and $\mathrm{L}_{1}$-norm results and the corresponding slope uncertainties were negligible for the subsequent analysis.

\section{Modeling Results}

We performed measurements of the teleseismic $P$-wave codas for each of the four velocity models. Coda traces were band-pass filtered to center frequencies of $0.4,0.79,1.58,3.16,4.48$, $6.32,8.94,12.6$, and $17.8 \mathrm{~Hz}$ to examine the log-amplitude slopes as functions of the frequency. Figure 6 shows the results for models Quartz and Complex Crust with crustal $Q_{S}=500$.

In Figure 6, coda slope values show clear linear dependencies on the frequency to about 7$10 \mathrm{~Hz}$, after which the fidelity of modeling appears to drop, and the slopes flatten out (attenuation is replaced by frequency-independent numerical noise of unclear nature). By fitting straight lines to the points within the $0-7 \mathrm{~Hz}$ range, we find that the intercepts at $f=0 \mathrm{~Hz}$ are non-zero: $\gamma \approx$ $0.8 \cdot 10^{-2}$ and $\gamma \approx 1.6 \cdot 10^{-2}$ for the two respective models. From the frequency dependencies of coda slopes, we measure $Q_{\mathrm{c}}$ (see equation 4 ), which equal $Q_{\mathrm{c}} \approx 800$ and 600, respectively (Figure 6). Note that $\gamma$ is clearly positive in all cases considered here, indicating that the geometrical attenuation is only partly compensated by the increasing volume of near-surface scattering. Also note the flattening of slope $(f)$ dependencies at higher frequencies due to the reduction in the signal/noise ratios. The outliers at lower frequencies should most likely be related to tuning effects within the crustal layers (Figure 6).

Alternatively, when directly converted to frequency-dependent $Q_{\text {coda }}(f)$ by using the conventional equation (2), the values of coda amplitude slopes could also be fit by using a dependence of type (1), with $Q_{0} \approx 250$ at $1 \mathrm{~Hz}$ and $\eta \approx 0.5$ (Figure 7). Although this interpretation could be acceptable within the synthetic or real data uncertainties, our model contains no rheological or frequency-selective scattering mechanisms to support an in situ frequency-dependent attenuation. Therefore the observed coda $Q_{\text {coda }}(f)$ (Figure 7) should better be viewed as an "apparent" quantity, or as an observational artifact.

By picking linear $\ln (A(t))$ dependencies (4) for each frequency, we found that the values of $\gamma$ stayed approximately constant in most cases that were close to the correct platform structure (values shown in labels in Figure 8). By taking advantage of this relative stability of $\gamma$ 's, we attempted directly relating the observed $Q_{\mathrm{c}}$ to the crustal $Q_{S}$. To accomplish this, we transformed the observed coda slopes into $Q_{\mathrm{c}} / Q_{S}$ ratios:

$$
\alpha\left(Q_{S}\right)=\frac{Q_{\mathrm{c}}}{Q_{S}}=\frac{\pi f}{-Q_{S}\left(\frac{d \ln A(t, f)}{d t}+\gamma\right)}
$$

for each frequency (Figure 8). Note that the resulting $\alpha\left(Q_{S}\right)=Q_{d} / Q_{S}$ ratios are nearly frequencyindependent, greater than 1, and approach 1 for higher $Q_{S}$ (Figure 8). Because of these properties, frequency-independent curves $\alpha\left(Q_{S}\right)$ can be approximated from numerical modeling in each model, as shown by lines in Figure 8. Once such curves are constructed by numerical modeling, they can be utilized for deriving the average crustal $Q_{S}$ from observations of $Q_{\mathrm{c}}$. Interestingly, the $\alpha\left(Q_{S}\right)$ functions are very similar (within $\sim 20 \%$ differences) for all three Quartz-based models, 
despite the strong difference in $\gamma$ for the Complex Crust model (Figure 8). However, the $\alpha\left(Q_{S}\right)$ curve for the IASP91 model (which is definitely inadequate for this area) is strongly different from those for Quartz in its shape, values, and stability in respect to frequency variations (Figure $8)$.

Finally, both $\gamma$ and the functional form of $\alpha\left(Q_{S}\right)$ should depend on the structures of the models. We tested this dependence by using four different types of models (2) and also by adding velocity perturbations to Quartz model. The results suggest that $\gamma$ and $\alpha\left(Q_{S}\right)$ indeed correlate with structural model styles and crustal velocities, as will be further elaborated on in Discussion.

\section{Discussion}

\section{$\alpha\left(Q_{s}\right)$ and modal content of coda wavefield}

In a heterogeneous lithosphere, areas of scattering and attenuation are unevenly distributed (likely most abundant closer to the surface), and the seismic wavefield is complex and far from any simple symmetry. Generally, crustal $S$ waves should be the primary contributors to the coda, and consequently we expect that the observed $Q_{c}$ is related to the crustal $S$-wave $Q_{S}$. However, the values of $Q_{s}$ and $Q_{\mathrm{c}}$ may differ. For our model, it appears that because a part of the seismic energy travels through the mantle with typically higher $Q_{S}$, we should expect values of $Q_{\mathrm{c}}$ to be greater than crustal $Q_{s}$. In regard to the geometrical attenuation parameter $\gamma$ in equation (4), it could be either negative (corresponding, for example, to strong reflected and diving waves returning additional energy to the surface) or positive, as observed in our modeling. Values of $\gamma>$ 0 correspond to the scattered energy decaying with distance faster than surface waves, which should be a common case due to: 1) high attenuation of surface waves in the weathered zone (note the Complex Crust model, Figure 8), and 2) multiple reflections and scattered seismic waves leaking into the mantle as suggested, for example, by Gupta et al. (1991).

Both $\gamma$ and the functional form of $\alpha\left(Q_{S}\right)$ depend on the velocity/density structures. A comparison of models Quartz and Complex Crust shows that coda decays are steeper (Figure 6) and $\alpha\left(Q_{S}\right)$ are lower (Figure 8) for Complex Crust. Both of these effects likely result from the presence of the low- $Q$ sedimentary layer in this model. Note that in a detailed study of PNE $L g Q$ in Russia, Li et al. (submitted to BSSA) pointed out a quantitative correlation of low $L g Q$ with the presence of young (low-velocity) sedimentary basins. From this correlation, a value of $Q_{S} \approx$ 60-70 was also estimated for these sedimentary rocks.

Interestingly, in the "realistic" crust/mantle models considered, $\alpha\left(Q_{S}\right)$ is not constant and decreases from $\sim 3-4$ at $Q_{S}=100$ to about $\sim 1-1.4$ at $Q_{S}=1000$ (Figure 8). As expected, the measured values of $\alpha\left(Q_{S}\right)$ are also fairly frequency-independent. By contrast, in the IASP91 model, $\alpha\left(Q_{S}\right)$ is roughly constant within $\sim 2.5-3.5$ range and somewhat less stable in respect to the frequency variations (Figure 8). This means that only in the IASP91 model, the observed $Q_{\mathrm{c}}$ is proportional to $Q_{S}$ of the crust, and it equals $Q_{\mathrm{c}}$ of the Quartz-based models only when $Q_{S} \approx 100$.

The above observations can be explained by considering two end-member types of waves contributing to the modeled codas (Figure 9). For the first type labeled C, propagation takes place predominantly through the crust, possibly with some contributions from mantle paths (Figure 9). For type M, only a fixed portion of the path lies within the crust. With these definitions, we observe that:

1) Waves propagating through the mantle, which generally has higher $Q_{S}$ values of $\sim 700-$ 1000 in our model, increase the observed values of $Q_{c}$. This effect is controlled by the relative time seismic waves travel within the mantle, and therefore waves of type $M$ 
should lead to higher $\alpha\left(Q_{S}\right)$ than those of type C (Figure 9);

2) Because of its simple crustal structure, the IASP91 model is depleted in crustal waves (diving and converted, Figure 3d), and therefore the contribution of scattering paths of type $\mathrm{M}$ in it is significant for any crustal $Q_{s}$. For such paths, $\alpha\left(Q_{S}\right)$ is determined by the time seismic waves travel within the crust, which stays approximately constant;

3) Waves of type $\mathrm{C}$ are significantly more abundant in the Quartz-based models (Figure 3ac). With increasing the crustal $Q_{s}$, their amplitudes relative to the wave type $M$ should increase, leading to the observed reduced values of $\alpha\left(Q_{S}\right)$. Note that $\alpha\left(Q_{S}\right)$ decreases with increased crustal gradients and reflectivity, and it reaches $\approx 1$ for the Complex Crust model (compare Figures $8 b$ to a);

4) For average crustal $Q_{s} \leq 100$, seismic waves dissipate quickly, and no significant crustal waves of type $\mathrm{C}$ should develop. Therefore, the results from all models should be similar for such $Q_{S}$ values. However, note that such low whole-crust $Q_{S}$ values are still unlikely, and they were not observed in PNE data (Li et al., submitted to BSSA).

\section{PNE data example}

The use of the $\left(\gamma, Q_{\mathrm{c}}\right)$ model allows us to resolve the following, hitherto puzzling observation from the PNE profiles. Within the East European Platform, measurements from Quartz profile resulted in $Q_{\text {coda }}(\sim 2 \mathrm{~Hz})=380$ and $Q_{\text {coda }}(\sim 5 \mathrm{~Hz}) \approx 430$, which was interpreted in the power-law form $(1)$ as $Q_{\text {coda }}(f) \approx 270 \cdot f^{0.3}$ (Morozov and Smithson, 2000). However, within the Siberian Craton (PNE Kimberlite-3), practically frequency-independent coda amplitude decays were observed, corresponding to $Q_{\text {coda }}(f) \approx 1050 \cdot f^{1.0}$ (Morozov et al., 2006). Although the intrinsic attenuation was expected to be low within the Siberian Craton, its strong frequency dependence is vastly different from the East European Platform and is surprisingly high even for tectonically active areas (Aki, 1980; Benz et al., 1997; Erickson et al., 2004).

The explanation of the above in the $\left(\gamma, Q_{c}\right)$ form is quite simple. From Quartz-4 data, the logarithms of coda amplitudes can be interpreted by using $\gamma \approx 0.75 \cdot 10^{-2} \mathrm{~s}^{-1}$ and frequencyindependent $Q_{\mathrm{c}} \approx 850$, with estimated range of uncertainty of $780-960$ (Figure 10). From Kimberlite-3 records, $\gamma$ turns out to be nearly the same, but $Q_{\mathrm{c}}$ is much higher: $Q_{\mathrm{c}} \approx 2500 \pm 300$ (Figure 10). Note that both values of $\gamma$ also agree remarkably well with the numerical simulations based on Quartz velocity model data above (Figure 6). The high values of $Q_{\mathrm{c}}$ within the Siberian Craton agree with observations of $P g$ waves propagating to over $1600 \mathrm{~km}$ from these PNEs (Morozov et al., 2006). These are likely the longest-propagating short-period $P g$ waves observed on Earth.

\section{Coda properties in stable vs. tectonically active regions}

Because the $\left(\gamma, Q_{c}\right)$ parameters appear more stable and directly related to lithospheric properties than $\left(Q_{0}, \eta\right)$, it would be useful to revisit the observation that active tectonic regions are generally characterized by low $Q_{0}$ and high $\eta$, and stable cratons - by higher $Q_{0}$ and lower $\eta$ (Aki, 1980; Steensma and Biswas, 1988; Benz et al., 1997; Mandal and Rastogi, 1998; Erickson et al., 2004). By using over 40 worldwide measurements, Morozov (in press) suggested that the level of $\gamma_{\mathrm{d}} \approx 0.8 \cdot 10^{-2} \mathrm{~s}^{-1}$ in surface-wave and $L g Q$ measurements unambiguously separates the tectonically stable $\left(\gamma<\gamma_{\mathrm{d}}\right)$ from tectonically active $\left(\gamma>\gamma_{\mathrm{d}}\right)$ zones. Note that both of the PNE coda examples above comply with this criterion. At the same time, the use of $Q_{0}$ or $Q_{\mathrm{c}}$ as discrimination criteria could be problematic because these quantities are strongly affected by sedimentary basins ( $\mathrm{Li}$ et al., submitted to BSSA) and generally vary quite broadly (Morozov, in 
press).

The reasons for increased $\gamma$ values within tectonically-active areas still remain to be investigated. In our numerical experiments, we tested two possible causes of higher geometrical attenuation: 1) lower-velocity, lower- or inverted-gradient crustal and upper mantle velocity structures, possibly caused by increased crustal temperatures (as in the Warm Quartz model; Christensen and Mooney, 1995), and 2) presence of a low-Q layer, as in the Complex Crust model (Figure 2). Both Quartz and Complex Crust models were examined with and without the low- $Q$ sedimentary layers, and the Quartz model was also tested for $\pm 5 \%$ velocity variations within the crust. These testing results suggested the following observations:

1) Velocity variations and/or reduced crustal velocity gradients have little effect on the values of $\gamma$ (Figure 7; also compare the results for Quartz and Warm Quartz models in Figure 8);

2) A low-Q layer within the upper crust causes $\gamma$ to increase (compare the Quartz and Complex Crust models; Figure 8).

Therefore, a thin, low- $Q$ or scattering layer within the crust most strongly affects the geometrical, but not the average intrinsic attenuation. This surprising at first glance conclusion can be explained by noting that the attenuation effect of the layer should be proportional not to the total number of wave cycles (from the source to receiver) but to the number of times the seismic waves traverse it during propagation, which is a geometrical factor. It is likely that a similar effect would be achieved with a low-Q layer located at different depths within the model, or with elastic scattering (e.g., from crustal faults or corrugated Moho) replacing the intrinsic attenuation of the sediments used in our modeling. However, these hypotheses still need to be developed in detailed case and modeling studies, which are outside of the scope of this paper.

\section{$\alpha\left(Q_{s}\right)$ and inversion for attenuation properties of the crust}

The stability of $\gamma$ and $\alpha\left(Q_{S}\right)$ in respect to the variations of crustal $Q$ provides a way for systematic inversion for the average crustal $Q_{s}$. First, from the available travel-time data (ideally, from inversion of a refraction/reflection profile similar to Quartz, travel-time tomography, or Receiver Function studies), a velocity/density model can be constrained. By using numerical modeling in such a structure, $\gamma$ and the $\alpha\left(Q_{S}\right)$ dependence can be derived as described above. With the help of these functions, bulk crustal $Q_{s}$ can then be estimated from $Q_{\mathrm{c}}$ observations:

$$
Q_{S}=\frac{Q_{\mathrm{c}}}{\alpha^{-1}\left(Q_{\mathrm{c}}\right)}
$$

where:

$$
Q_{c}=\frac{\pi f}{-\left(\frac{d \ln A(t, f)}{d t}+\gamma\right)},
$$

which should be frequency-independent. The inversion scheme (11-12) is further elaborated and applied to PNE profiling records in Part II of this paper. In addition, because parameters $\gamma$ and the in situ $Q_{s}$ in equation (11) are related to the crustal structure and not so much to the observations, their use could improve coda magnitude analysis and calibration (Phillips et al., 2004) and coda $Q$ regionalization (Singh and Herrmann, 1983).

The scattering potential $\Psi$ in integral (9) is not constrained in our modeling and is not 
critical for the results presented above. However, it still was also estimated to match the observed PNE data. Morozov and Smithson (2000) modeled coda power as a function of time by describing the primary event as a parabolic function with peak power $P^{0}$ and duration $\tau$, and the relative coda amplitude parameter $\lambda$, with the product $\lambda \tau$ giving the power relative to the peak. These authors determined values of $\lambda=0.22$ for all Quartz events and $\tau=1.25 \mathrm{~s}$ for the teleseismic $P$. Applying these values to the synthetic data in this study gives a scatteredamplitude to direct-amplitude ratio of $\sqrt{\lambda \tau} \approx 0.5$. Therefore, the scattered trace should be approximately half the amplitude (and about $1 / 4$ the intensity) of the direct wave at the time of coda onset. From this scaling factor, the scattering potential $\Psi$ per unit area was computed for its use in equation (9).

With regard to the validity of the single-scattering coda approximation (eq. 9), note that the intensity of the doubly-scattered coda should be approximately $1 / 4$ of that of the singlescattered coda considered above. Therefore, we expect that accounting for multiple scattering within the coda could correct the coda intensity by roughly $(1 / 4) /(1-1 / 4)=1 / 3$. The empirical calibration of the value of $\Psi$ above by using the real PNE data (which is multiply-scattered by nature) should further reduce this error. Therefore, among the other limitations of the present modeling (1D velocity structure, absence of surface topography, only surface scattering, and frequency- and spatially invariant $\Psi$ ), this appears to be a reasonable approximation. Moreover, the multiply-scattered coda contributions should likely have similar time-frequency properties, which were the main object of this study.

\section{Conclusions}

Frequency-dependent attenuation often observed in local, regional, and teleseismic coda measurements, as well as in surface-wave, $L g$, and borehole studies. Such observations can be interpreted in two ways: via frequency-dependent coda $Q(f)$ or by means of effective, uncompensated geometrical attenuation ( $\gamma$, comprising uncompensated geometrical spreading and elastic scattering) and frequency-independent coda attenuation $\left(Q_{c}\right)$. The second of these alternatives offers a number of advantages:

1) It is better justified theoretically and does not require unusual crustal rheologies or scalelength selective scattering properties.

2) It can be measured directly and unambiguously from the data, without reliance on compensation of geometrical spreading or other theoretical assumptions.

3) Its parameters can be quantitatively related to the in situ lithospheric velocity/density structure (via $\gamma$ ) and attenuation (via $Q_{c}$ ) crustal structures. The resulting $Q_{\mathrm{c}}$ appears to correspond to crustal $Q_{S}$ and can be directly correlated to the observed $L g Q$.

4) The stable, frequency-independent parameters allow easier comparisons between different areas and studies around the world. In particular, parameter $\gamma$ could provide a transportable discriminant for differentiating between the tectonic types and ages of the lithosphere. From a preliminary numerical model, this discriminant may indicate the presence of localized attenuative or scattering structures within the crust.

5) As stable and transportable characteristics, the $\left(\gamma, Q_{c}\right)$ parameters are likely to improve coda magnitude analysis and coda $Q$ regionalization.

Numerical modeling of PNE coda wavefields argues strongly in favor of the $\left(\gamma, Q_{c}\right)$ model and illustrates the mechanisms leading to apparent frequency dependencies of coda $Q(f)$ that are often reported. PNE coda measurements in two different areas in Russia show similar $\gamma$ 
values although strongly different $Q_{\mathrm{c}}$. Remarkably, the observed values of $\gamma$ are found to be very close to those derived from totally independent numerical simulations.

\section{Acknowledgements}

We thank Dr. Anton Dainty for his advice and for pointing out his earlier paper (Dainty, 1981). Numerous comments in reviews by Drs. N. Biswas and K. Mackey greatly helped in improving the manuscript. This work was supported by DTRA Grant DTRA01-01-C-0057, NNSA Grant DE-FC52-05NA26609, and also in part by Canada NSERC Discovery Grant RGPIN261610-03.

\section{Data and Resources}

The nuclear explosion data used in this paper were acquired by the Deep Seismic Sounding program in the former Soviet Union in $1977-84$. These records are currently available through IRIS archives (http://www.iris.edu). Modeling and data analysis were performed using the IGeoS package (Morozov, 2008; http://seisweb.usask.ca/igeos). GMT programs (Wessel and Smith, 1995) were used for making PostScript plots. 


\section{References}

Abercrombie, R. E. (1998). A summary of attenuation measurements from borehole recordings of earthquakes: the $10 \mathrm{~Hz}$ transition problem, Pageoph, 153, 475-487.

Adams, D. A., and R. E. Abercrombie (1998). Seismic attenuation above $10 \mathrm{~Hz}$ in southern California from coda waves recorded in the Cajon pass borehole, J. Geophys. Res. 103, 24,257-24,270.

Aki, K. (1980). Scattering and attenuation of shear waves in the lithosphere, J. Geophys. Res. 85, 64966504.

Aki, K. and B. Chouet (1975). Origin of coda waves: source, attenuation, and scattering effects, $J$. Geophys. Res. 80, 3,322-3,342.

Aki, K., and Richards, P. G. (2002). Quantitative Seismology, Second Edition, University Science Books, Sausalito, CA.

Aster, R., B. Borchers, and C. Thurber (2002). Parameter Estimation and Inverse Problems, preliminary edition.

Bannister, S. G., E. S. Husebye, and B. O. Ruud (1990). Teleseismic $P$ coda analyzed by three-component and array techniques: deterministic location of topographic $P$-to- $R g$ scattering near the NORESS array, Bull. Seism. Soc. Am. 80, 1969-1986.

Benz, H., A. Frankel, and D. Boore (1997). Regional Lg attenuation in the continental United States, Bull. Seism. Soc. Am. 87, 600-619.

Campillo, M. (1987). $L g$ wave propagation in a laterally varying crust and the distribution of the apparent quality factor in central France, J. Geophys. Res. 92, 12604-12614.

Campillo, M. (1990). Propagation and attenuation characteristics of the crustal phase Lg, Pageoph, 132, 117.

Christensen, N. I., and W. D. Mooney (1995). Seismic velocity structure and composition of the continental crust: A global view, J. Geophys. Res. 100, B7, 9761-9788.

Chubak, G., and I. B. Morozov (2006). Integrated software framework for processing of geophysical data, Computers \& Geosciences, 32, 767-775.

Dainty, A. M. (1981). A scattering model to explain seismic Q observations in the lithosphere between 1 and $30 \mathrm{~Hz}$, Geophys. Res. Lett. 8, 1126-1128.

Dainty, A. M. (1985). Air Force Geophysical Laboratory Report, AFGL-TF-86-0218.

Dainty, A. M. (1990). Studies of coda using array and three-component processing, Pageoph. 132, 221244.

Dainty, A. M. and C. A. Schultz (1995). Crustal reflections and the nature of regional $P$ coda, Bull. Seismol. Soc. Am. 85, 851-858.

Der, Z. A., A. C. Lees, and V. F. Cormier (1986). Frequency dependence of $Q$ in the mantle underlying the shield region of Eurasia, Part III: The Q-model, Geophys. J. R. Astr. Soc. 87, 1103-1112.

Enderle, U., M. Titgemeyer, M. Itzin, C. Prodehl, and K. Fuchs (1997). Scales of structure in the lithosphere - Images of processes, Tectonophysics 275, 165-198.

Erickson, D., D. E. McNamara, and H. Benz (2005). Frequency-dependent Lg Q within the continental United States, Bull. Seism. Soc. Am. 94, 1630-1643.

Frankel, A., A. McGarr, J. Bicknell, J. Mori, L. Seeber, and E. Cranswick (1990). Attenuation of highfrequency shear waves in the crust: measurements from New York state, South Africa, and southern California, J. Geophys. Res. 95, 17441-17457. 
Fuchs, K., and G. Müller (1971). Computation of synthetic seismograms with the reflectivity method and comparison with observations, J. R. Astronom. Soc. 23, 417-433.

Greenfield, R. J. (1971). Short-period P-wave generation by Rayleigh-wave scattering at Novaya Zemlya, J. Geophys. Res. 76, 7988-8002.

Gupta, I.N., T. W. McElfresh, and R. A. Wagner (1991). Near-source scattering of Rayleigh to $P$ in teleseismic arrivals from Pahute Mesa (NTS shots, in: Taylor, S. R., H. J. Patton, and P. G. Richards (Eds.), Explosion Source Phenomenology, AGU Geophys. Monograph, 65, 151 - 160.

Hasegawa, H. S. (1985). Attenuation of $L_{g}$ waves in the Canadian Shield, Bull. Seism. Soc. Am. 75, 15691582.

Kennett, B. L. N. and R. Engdahl (1991). Traveltimes for global earthquake location and phase identification, Geophys. J. Int. 105, 429-465.

Kinoshita, S. (1994). Frequency-dependent attenuation of shear waves in the crust of the southern Kanto area, Japan, Bull. Seismol. Soc. Am. 84, 1387-1396.

Li, H., I. B. Morozov, and S. B. Smithson (submitted to Bull. Seism. Soc. Am.) Mapping $L g Q$ at 1-3 Hz in Northern Eurasia using nuclear-explosion profiles, manuscript available from: http://seisweb.usask.ca/downloads/Papers/LGQ/

Liu, H. P., Anderson, D. L., Kanamori, H. (1976). Velocity dispersion due to inelasticity: implications for seismology and mantle composition, Geophys. J. R. Astr. Soc. 47, 41-58.

Mandal, P., and B. K. Rastogi (1998). A frequency-dependent relation of coda $Q_{c}$ for Koyna-Warna region, India, Pageoph 153, 163-177.

Mayeda, K., and W. R. Walter (1996). Moment, energy, stress drop, and source spectra of western United States earthquakes from regional coda envelopes, J. Geophys. Res. 101, 11195-11208.

McNamara, D. E. (2000). Frequency-dependent Lg attenuation in south-central Alaska, Geophys. Res. Lett. 27, 3949-3952.

McNamara, D. E., T. J. Owens, and W. R. Walter (1996). Propagation characteristics of $L g$ across the Tibetian Plateau, Bull. Seism. Soc. Am. 86, 457-469.

Mechie, J., A. V. Egorkin, K. Fuchs, T. Ryberg, L. Solodilov, and F. Wenzel (1993). P-wave velocity structure beneath northern Eurasia from long-range recordings along the profile Quartz, Phys. Earth Planet Inter. 79, 269-286.

Mitchell, B. J. and L. Cong (1998). $L g$ coda $Q$ and its relation to the structure and evolution of continents: a global Perspective, Pegeoph. 153: 655-663.

Mitchell, B. J., Pan, Y., Xie, J., Cong, L. (1997). $L g$ coda $Q$ variation across Eurasia and its relation to crustal evolution, J. Geophys. Res. 102: 22767-22779.

Morozov, I. B. (2001). Comment on "High-frequency wave propagation in the uppermost mantle" by T. Ryberg and F. Wenzel, J. Geophys. Res. 106, 30,715-30,718.

Morozov, I. B. (2008). Open-source software framework integrates data analysis, EOS, Trans. Am. Geophys. Union, 89, 261-262.

Morozov, I. B. (in press). Geometrical attenuation, frequency dependence of $Q$, and the absorption band problem, Geophys. J. Int.

Morozov, I. B., and S. B. Smithson (2000).Coda of long-range arrivals from nuclear explosions, Bull. Seism. Soc. Am. 90, 929-939.

Morozov, I. B., E. A. Morozova, and S. B. Smithson (1998a). On the nature of the teleseismic Pn phase observed in the recordings from the ultra-long profile "Quartz", Russia, Bull. Seism. Soc. Am. 88, $62-73$. 
Morozov, I. B., E. A. Morozova, S. B. Smithson, and L. N. Solodilov (1998b). 2-D image of seismic attenuation beneath the Deep Seismic Sounding profile "Quartz", Russia, Pageoph. 153, 311-348.

Morozov, I., Morozova, E., Zhang, C. Chubak, G., Phillips, W.S. and Lipovetsky, I. (2006). Magnitudeyield and travel-time calibration of northern Eurasia using deep seismic sounding datasets, 28-th NNSA/DOE Seismic Research Review, Orlando, FL, September 2006.

Morozova, E. A., I. B. Morozov, S. B. Smithson., and L. N. Solodilov (1999). Heterogeneity of the uppermost mantle beneath Russian Eurasia from the ultra-long range profile QUARTZ, $J$. Geophys. Res. 104 (B9), 20,329-20,348.

Nuttli, O. W. (1973). Seismic wave attenuation and magnitude relations for eastern North America, $J$. Geophys. Res. 78, 5212-5218.

Padhy, S. (2005). A scattering model for seismic attenuation and its global application, Phys. Earth Planet. Int., 148, 1-12.

Phillips, W.S., H.J. Patton, S.R. Taylor, H.E. Hartse, and R. E. Randall (2004). Calibration for coda based magnitude and yield, 26th Seismic Research Review, 449-456, Orlando, Florida, September 2123.

Ryberg, T., and F. Wenzel (1999). High-frequency wave propagation in the uppermost mantle, J. Geophys. Res. 104, 10,655-10,666.

Ryberg, T., K. Fuchs, A. V. Egorkin, and L. Solodilov (1995). Observations of high-frequency teleseismic Pn on the long-range Quartz profile across northern Eurasia, J. Geophys. Res. 100, 18151-18163.

Sato, H. (1990). Unified approach to amplitude attenuation and coda excitation in the randomly inhomogeneous lithosphere, Pageoph. 132, 1-29.

Sato, H., and M. Fehler (1998). Seismic Wave Propagation and Scattering in the Heterogeneous Earth, Springer-Verlag, New York.

Singh, S., and R. B. Herrmann (1983). Regionalization of crustal coda $Q$ in the Continental United States, J. Geophys. Res. 88, 527-538.

Steensma, G. J., and N. N. Biswas (1988). Frequency Dependent Characteristics of Coda Wave Quality Factor in Central and South-central Alaska, Pageoph 128, 295-307.

Warren, N. (1972). Q and structure, Earth, Moon, and Planets, 4 (3-4), 430-441, Springer Netherlands.

Wessel P., and W. H. F. Smith (1995). New version of the Generic Mapping Tools released, EOS, Trans. Am. Geophys. Union, 76, 329. 


\section{Figures}

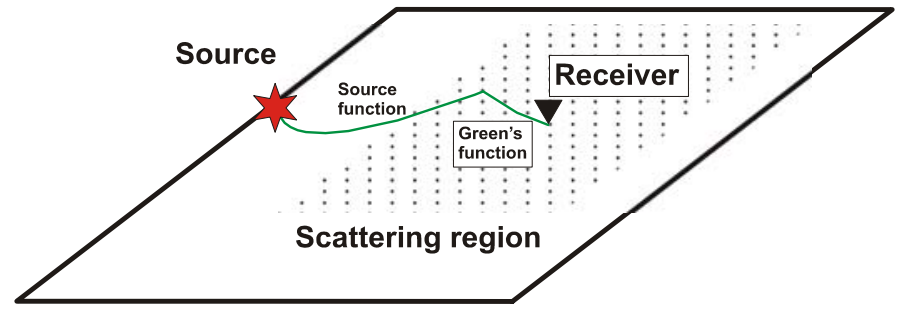

Figure 1. Surface scattering model (eq. 7). Seismic energy originates at the source, scatters from randomly and uniformly distributed surface points, and is detected at the receiver. The numerically-simulated Source and Green's functions describe the propagation of seismic energy.
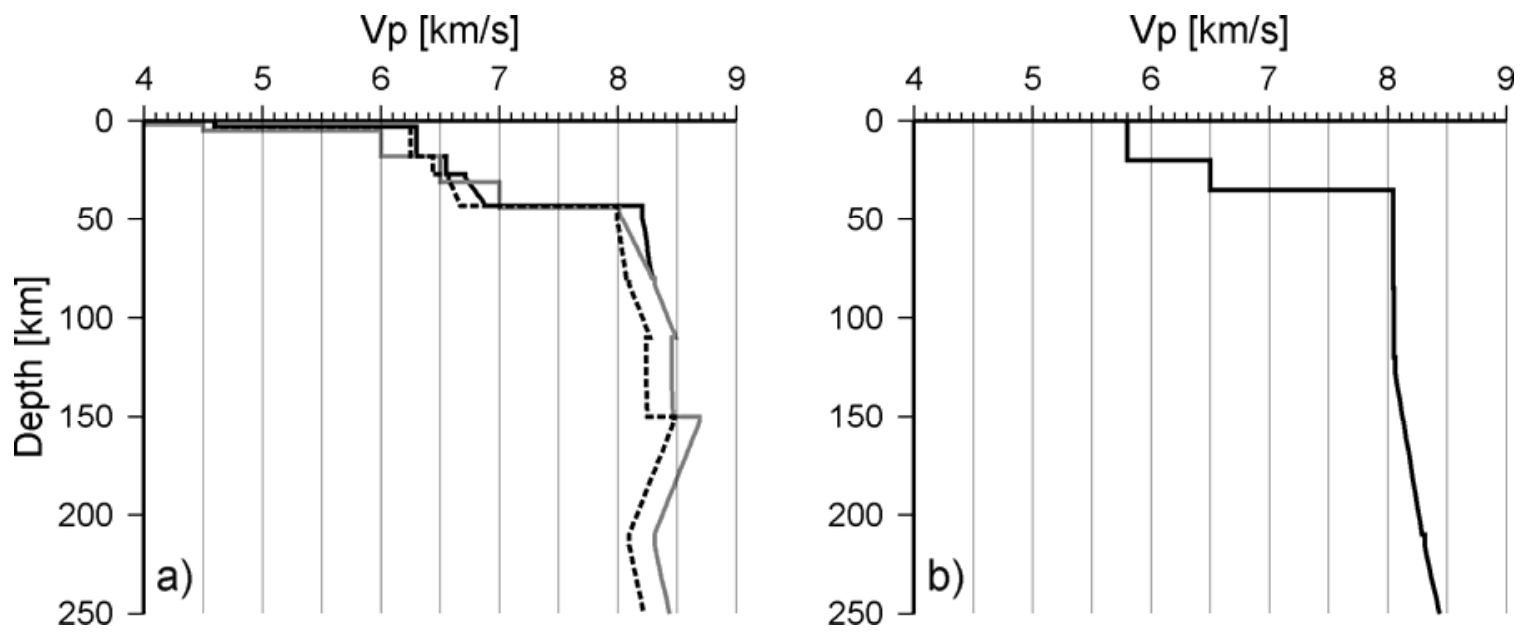

Figure 2. Upper $250 \mathrm{~km}$ of 1-D $V_{P}$ velocity models used in creation of synthetic seismograms. a) Models based on PNE Quartz results by Morozova et. al. (1999): Quartz (solid black line), Warm Quartz (dotted black), and Complex Crust (gray); b) IASP91 model. 


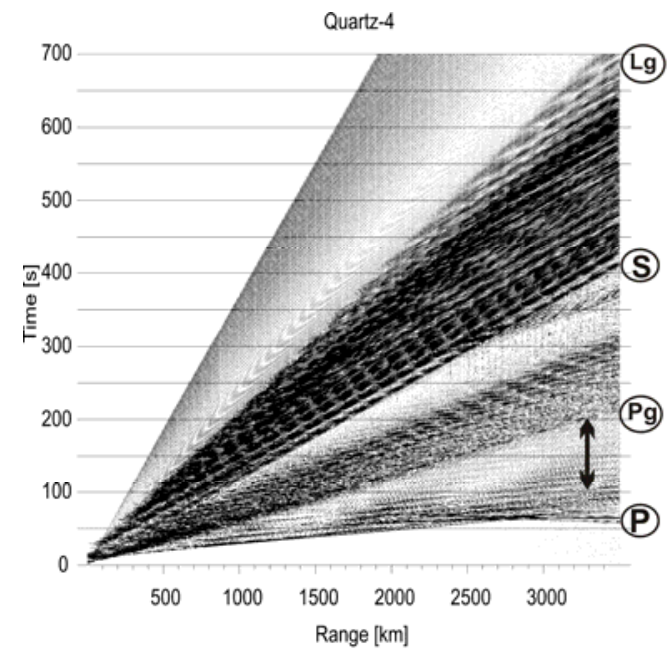

(A)

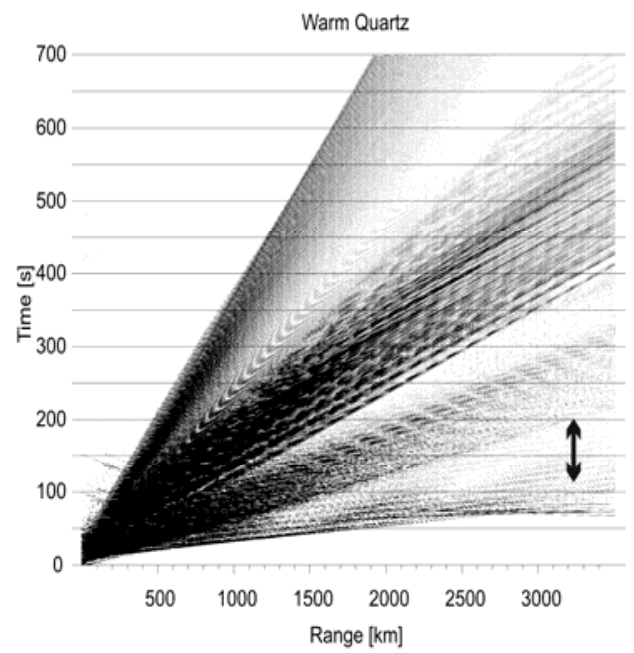

(C)

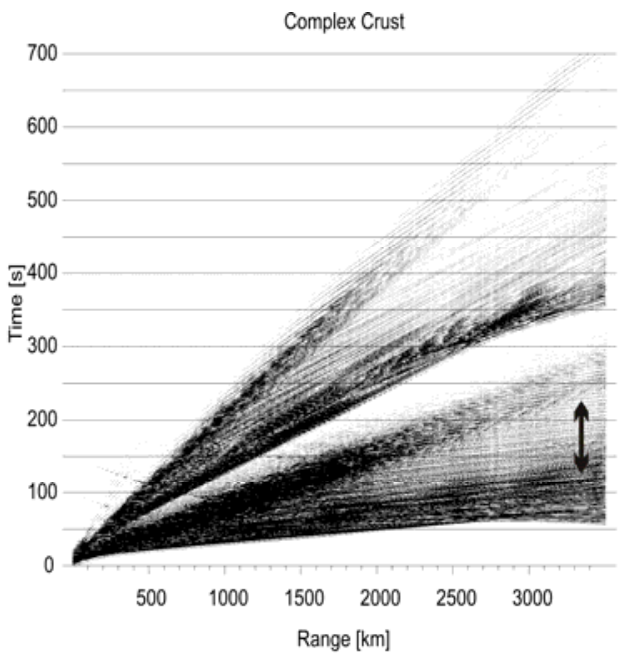

(B)

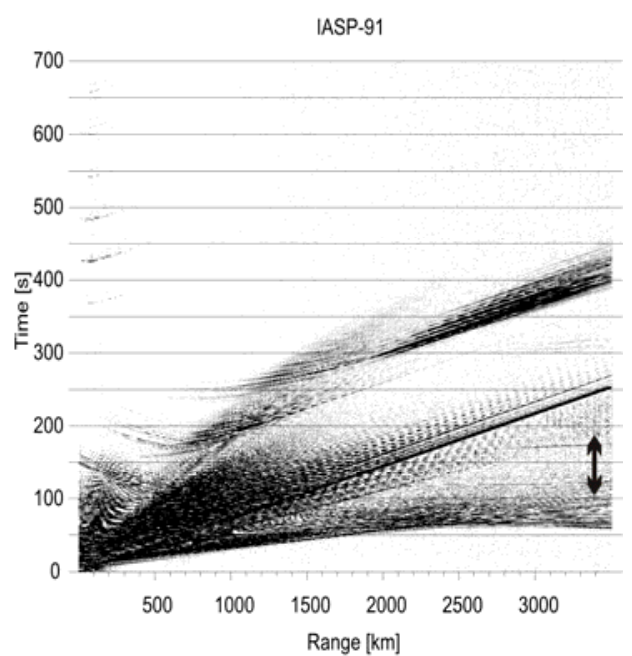

(D)

Figure 3. Reflection synthetic seismograms used as Green's functions, created using the four different velocity models in Figure 2: a) Quartz-4, b) Complex Crust, c) Warm Quartz, and d) IASP91. Note the similarities between wavefields a-c), and a contrast with d). Arrows indicate the positions of the receiver and coda time ranges used in the analysis. The key regional phases are labeled. 


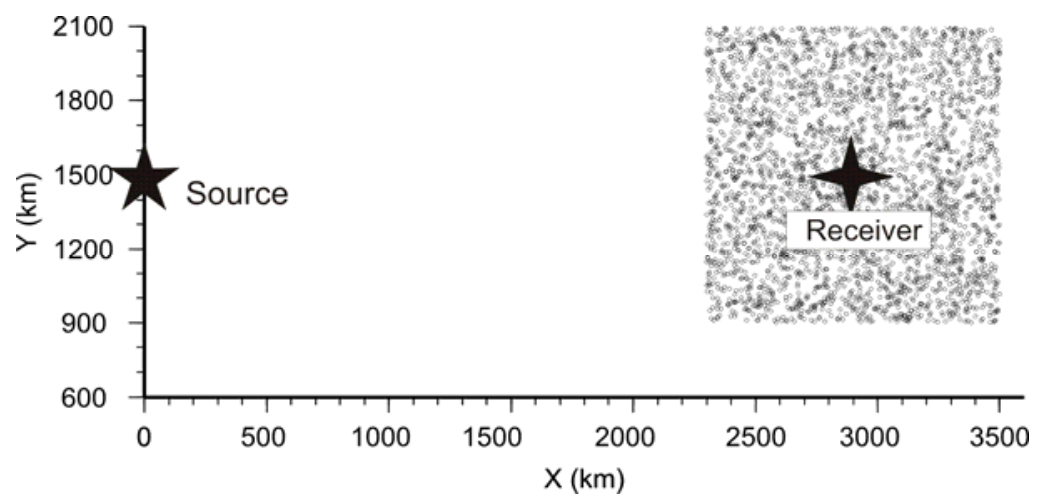

Figure 4. Scattering geometry. Scatterer locations within the region centered on the receiver are uniformly and randomly distributed.

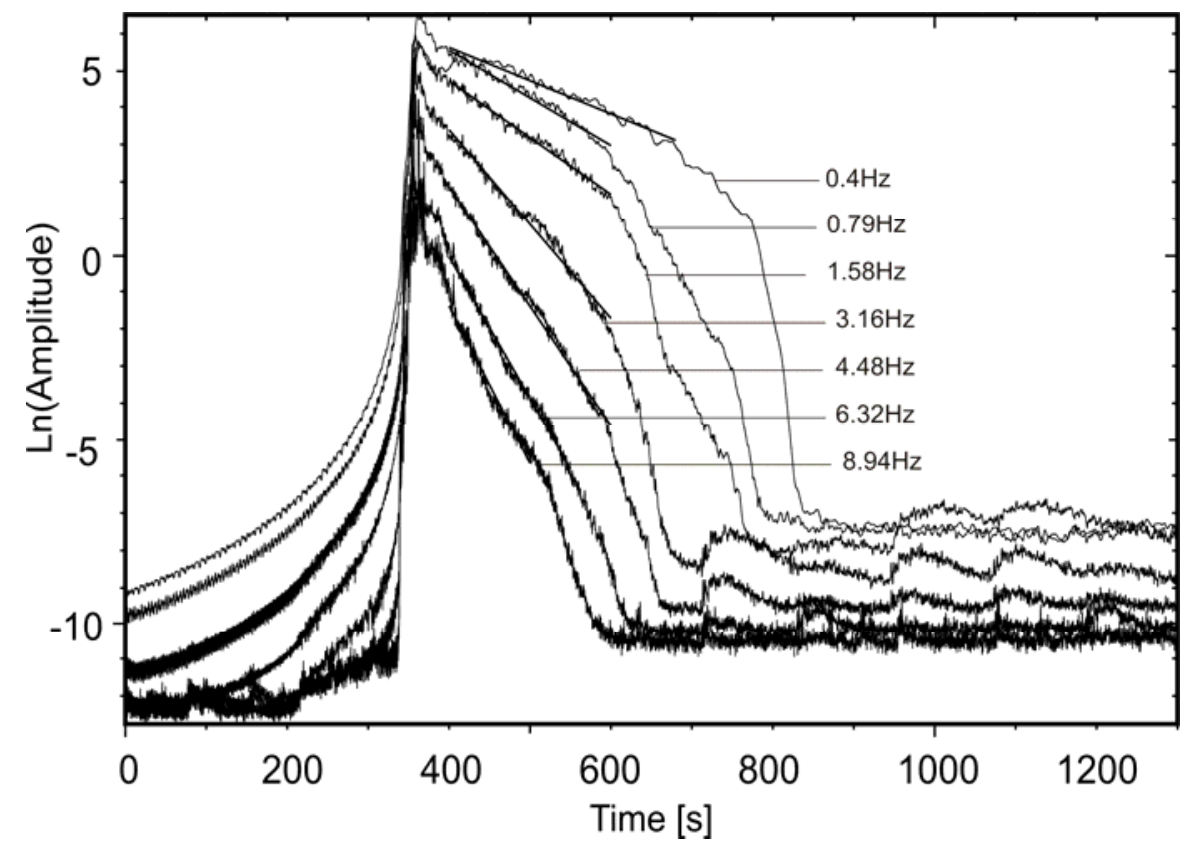

Figure 5. Log-amplitude synthetic coda for Quartz model (Figure 2a) at selected frequencies (labeled). Straight lines indicate the measured amplitude fits. Note the progressive change in coda shapes with increasing frequencies. 

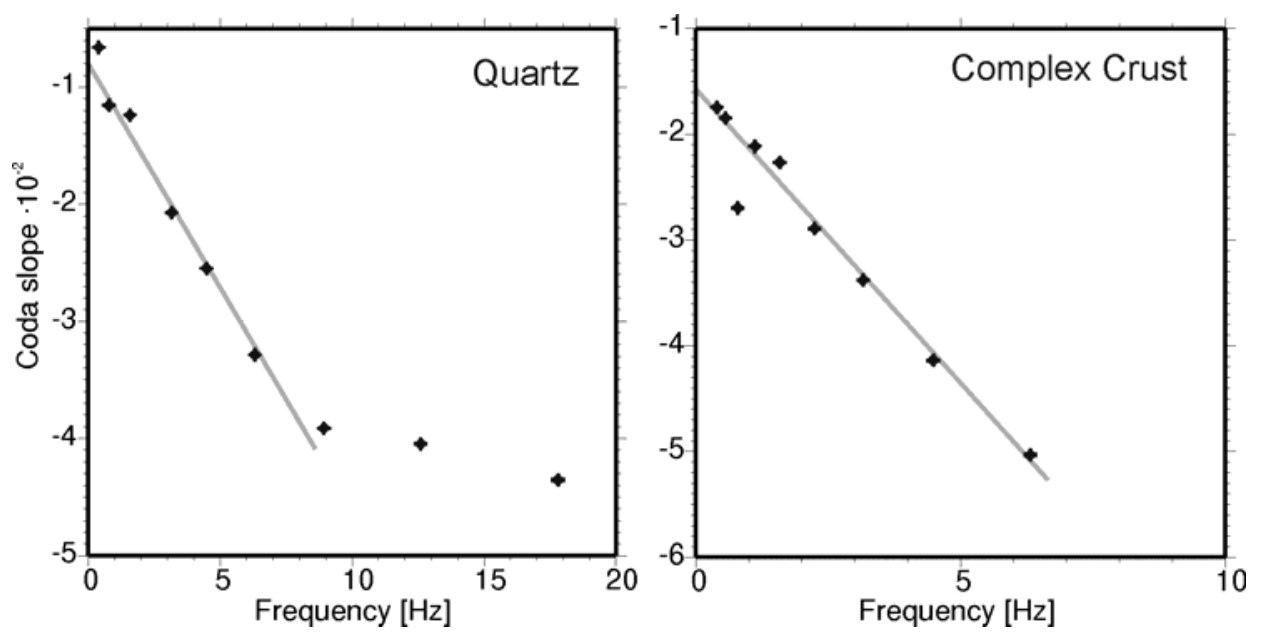

Figure 6. Measured log-amplitude coda slopes from models Quartz and Complex Crust with crustal $Q_{S}=500$. Note the linearly decreasing (steepening) coda slopes with frequencies increasing to $\sim 7-10 \mathrm{~Hz}$. Solid gray lines indicate linear regressions using formula (4): $\gamma=$ $0.8 \cdot 10^{-2}$ and $Q_{\mathrm{c}}=800$ for Quartz, and $\gamma=1.6 \cdot 10^{-2}$ and $Q_{\mathrm{c}}=600$ for Complex Crust models.

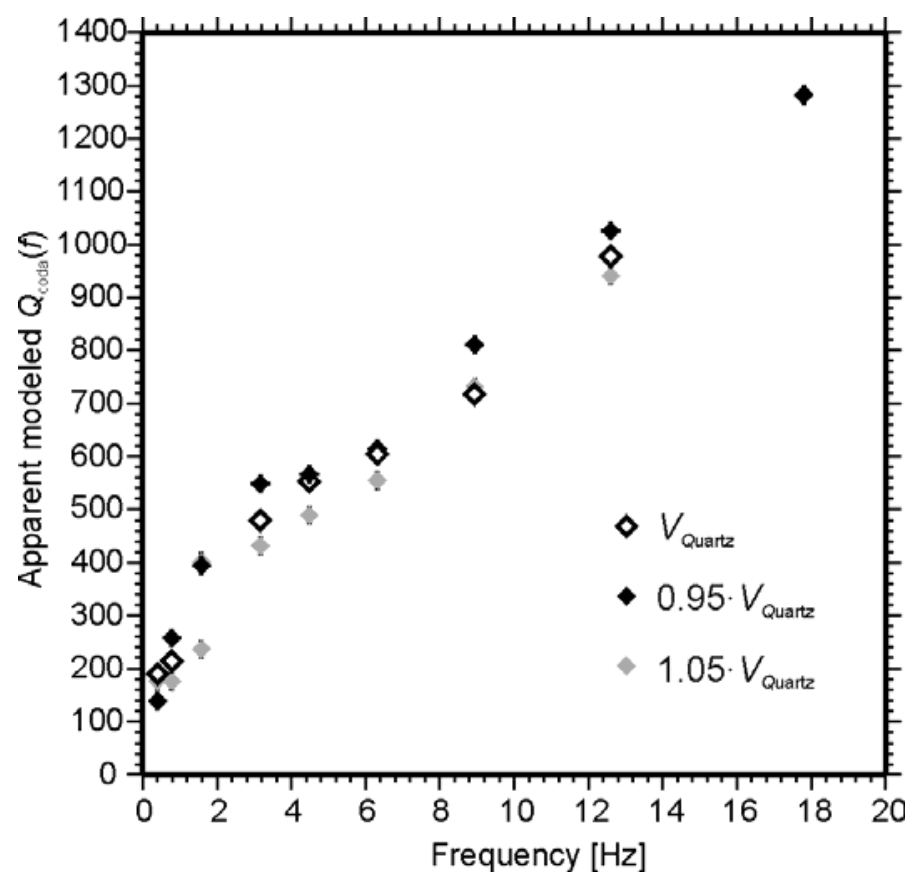

Figure 7. Measured log-amplitude coda slopes (Figure 6) transformed to apparent coda $Q(f)$ determined o relation (2). Note the strong frequency dependence $(\eta \approx 0.5)$ of $Q(f)$ despite the constant- $Q_{S}$, Newtonian modeled crustal rheology. 

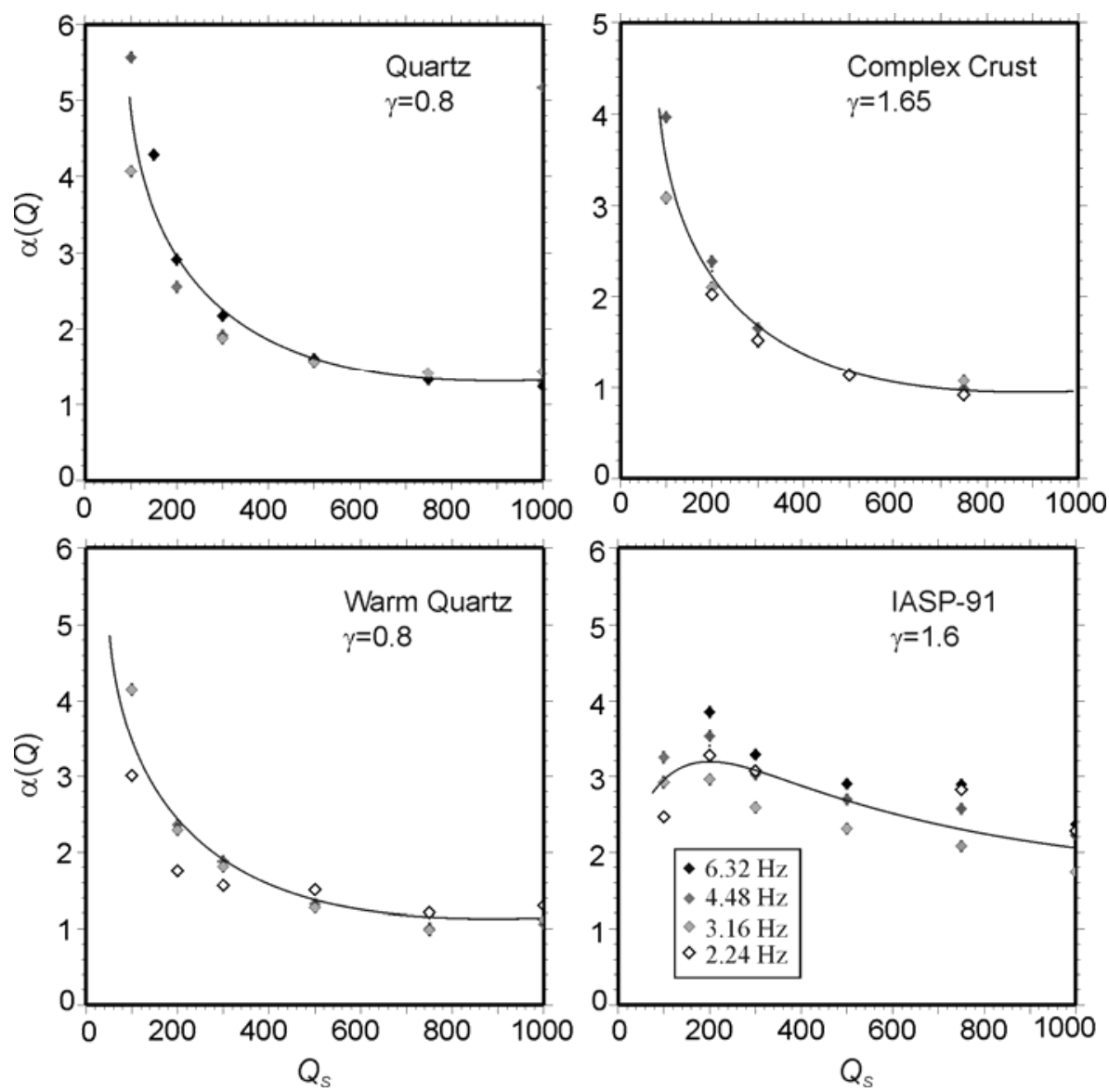

Figure 8. $\alpha\left(Q_{s}\right)$ from equation (10) for the four models of this study (labeled) and different frequencies (labeled symbols), obtained directly from coda slopes (Figure 6) by using the values of $\gamma$ in equation (4) shown in the labels. Lines represent the empirical $\alpha\left(Q_{S}\right)$ dependencies for each velocity structure, picked by hand.

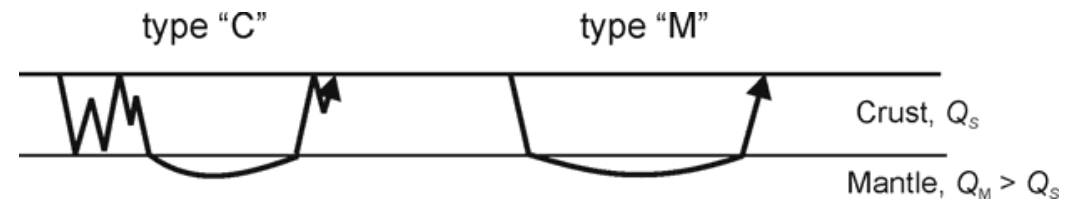

Figure 9. Two end-member types of scattered wave paths contributing to the coda. Type C: predominantly crustal propagation with numerous bounces between crustal boundaries. Type M: paths with at least a proportional contribution from mantle propagation. In all cases, $Q_{\mathrm{c}}>Q_{\mathrm{s}}$; however, modes of type C result in $Q_{\mathrm{c}} \approx Q_{s}$. Type M leads to increased values of $Q_{\mathrm{c}}$ reaching about $3 Q_{S}$ for the lower values of $Q_{S} \approx 100$ in our simulations (Figure 8). 


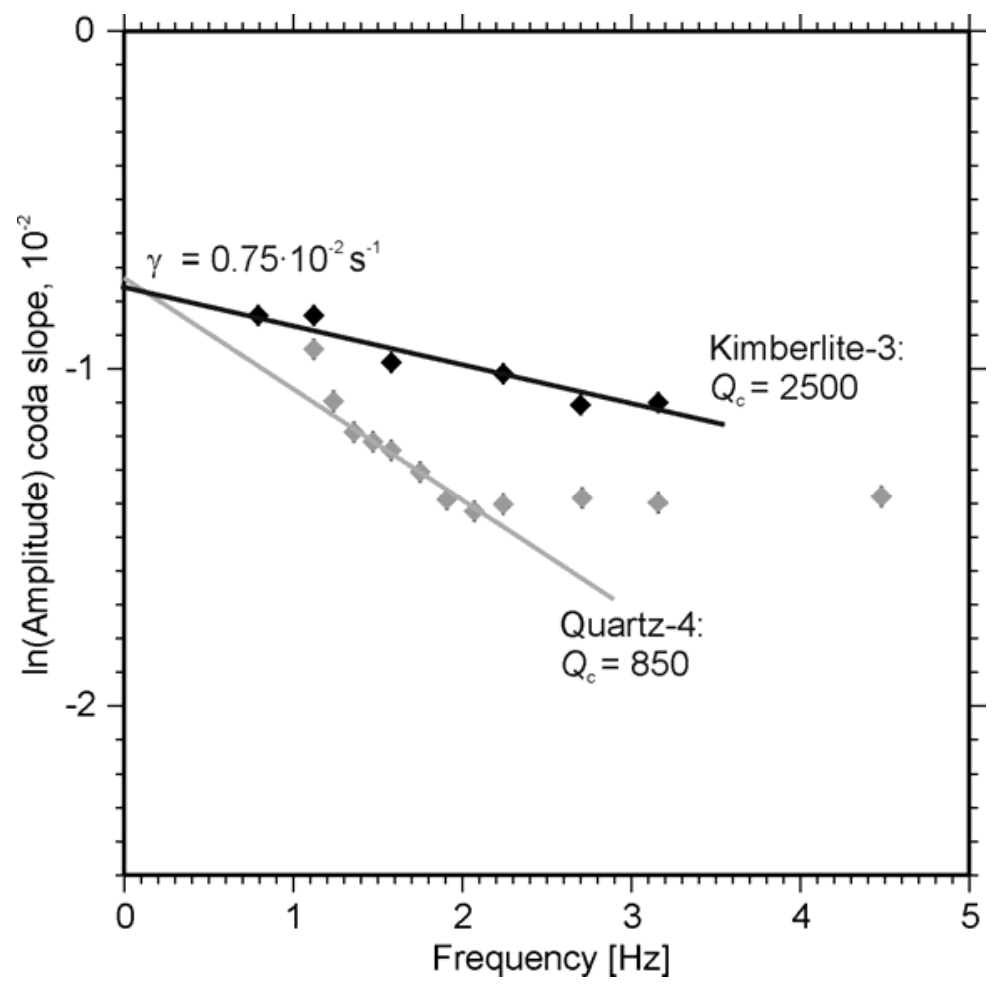

Figure 10.Log-amplitude coda slopes in real data from PNEs Quartz-4 (recorded within the EastEuropean Platform, at $2600-\mathrm{km}$ source-receiver offset; same data as in Morozov et al., 2002) and Kimberlite-3 (recording within the Siberian Craton at $1100-\mathrm{km}$ offsets). Note the different $Q_{\mathrm{c}}$ values (lines and labels), whereas the $\gamma$ values are similar and close to those modeled (Figure 6). Also note that because of lower-magnitude source and longer recording distance, the usable frequency band is narrower for Quartz PNE records. 